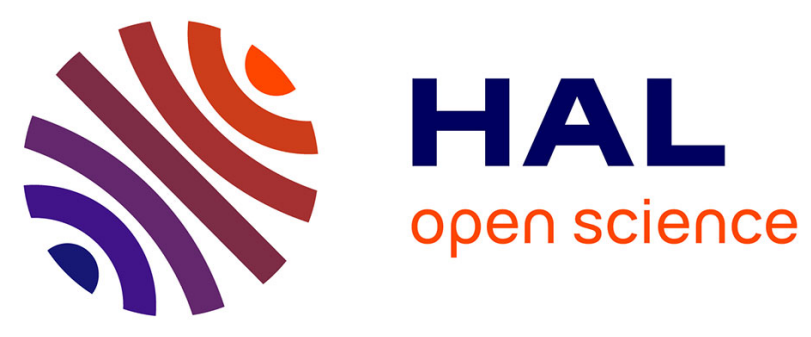

\title{
Monolithic Free-Standing Large-Area Vertical III-N Light-Emitting Diode Arrays by One-Step h-BN-Based Thermomechanical Self-Lift-Off and Transfer
}

Soufiane Karrakchou, Suresh Sundaram, Rajat Gujrati, Phuong Vuong, Adama Mballo, Hibat Adjmi, Vishnu Ottapilakkal, Walid El Huni, Karim Bouzid, Gilles Patriarche, et al.

\section{To cite this version:}

Soufiane Karrakchou, Suresh Sundaram, Rajat Gujrati, Phuong Vuong, Adama Mballo, et al.. Monolithic Free-Standing Large-Area Vertical III-N Light-Emitting Diode Arrays by One-Step h-BN-Based Thermomechanical Self-Lift-Off and Transfer. ACS Applied Electronic Materials, 2021, 3 (6), pp.26142621. 10.1021/acsaelm.1c00206 . hal-03350389

\author{
HAL Id: hal-03350389 \\ https://hal.science/hal-03350389
}

Submitted on 24 Dec 2021

HAL is a multi-disciplinary open access archive for the deposit and dissemination of scientific research documents, whether they are published or not. The documents may come from teaching and research institutions in France or abroad, or from public or private research centers.
L'archive ouverte pluridisciplinaire HAL, est destinée au dépôt et à la diffusion de documents scientifiques de niveau recherche, publiés ou non, émanant des établissements d'enseignement et de recherche français ou étrangers, des laboratoires publics ou privés. 


\section{Monolithic Free-Standing Large-Area Vertical III-N}

\section{Light Emitting Diode Arrays by One-Step h-BN-based}

\section{Thermo-Mechanical Self-Lift-Off and Transfer}

Soufiane Karrakchou ${ }^{1,2}$, Suresh Sundaram ${ }^{1,2,3}$, Rajat Gujrati ${ }^{2,4}$, Phuong Vuong ${ }^{2}$, Adama Mballo², Hibat

E. Adjmi ${ }^{2}$, Vishnu Ottapilakkal ${ }^{2}$, Walid El Huni ${ }^{5}$, Karim Bouzid ${ }^{5}$, Gilles Patriarche ${ }^{6}$, Ali Ahaitouf ${ }^{3, \dagger}$,

Paul L. Voss ${ }^{1,2}$, Jean Paul Salvestrini ${ }^{1,2,3, *}$, Abdallah Ougazzaden ${ }^{1,2}$

${ }^{1}$ Georgia Institute of Technology, School of Electrical and Computer Engineering, Atlanta, GA 30332 0250, USA,

${ }^{2}$ CNRS, UMI 2958 Georgia Tech - CNRS, 2 rue Marconi, 57070 Metz, France,

${ }^{3}$ Georgia Tech Lorraine, 2 rue Marconi, 57070 Metz, France,

${ }^{4}$ Georgia Institute of Technology, Woodruff School of Mechanical Engineering, Atlanta, GA 303320250, USA

${ }^{5}$ Institut Lafayette, 2 rue Marconi, 57070 Metz, France.

${ }^{6}$ Centre de Nanosciences et de Nanotechnologies, Université Paris-Saclay, C2N-Site de Marcoussis, Route de Nozay, 91460 Marcoussis, France.

KEYWORDS: Vertical thin-film LEDs, 2D h-BN, Thermo-mechanical self-lift-off and transfer, van der Waals epitaxy, Free-standing III-N membranes, Flexible III-N optoelectronics. 
ABSTRACT. We demonstrate the fabrication of vertical InGaN light emitting diodes on large-area freestanding membranes, using a mechanical lift-off technique enabled by $2 \mathrm{D}$ h-BN. $30-\mu \mathrm{m}$-thick electroplated copper deposited on the epilayer (i) gives rigidity to the structure, preventing crack generation, (ii) functions as a back mirror and as a heat sink, and (iii) enables one-step self-lift-off and transfer of LED structures from h-BN/sapphire during a thermal treatment at $100^{\circ} \mathrm{C}$. Free-standing arrays of LEDs on thick membranes were processed and their electro-optical performance characterized. This approach can provide a solution for the fabrication of low cost, wafer scale, crack-free, and highly reproducible free-standing arrays of vertical LEDs with up to centimeter-size areas.

\section{Introduction}

In the recent years, innovation in high-brightness GaN-based light-emitting diodes (LEDs) has enabled the next-generation solid-state lighting, displays and visible light communication systems ${ }^{1}$. However, conventional laterally contacted LEDs grown on insulating sapphire substrates suffer from (i) high series resistance caused by lateral current path and by current crowding near the edge of the contact ${ }^{2,3}$, (ii) poor heat dissipation when operated at high current density due to the low thermal conductivity of the substrate $^{4}$, and (iii) limited top surface emission owing to the extensive coupling of light in the thick transparent sapphire substrate ${ }^{5,6}$. To overcome these issues, vertical thin-film LEDs (VTF-LEDs), where the sapphire substrate is removed and the LED structure is transferred to an electrically conductive host substrate, have been widely investigated. In this configuration, electrical contacts are deposited on both sides of the functional epilayers. VTF-LEDs where shown to exhibit lower series resistance and operation voltage due to the vertical current injection ${ }^{7,8}$. Moreover, transfer to metallic substrates allows for efficient heat extraction when LEDs are driven at high current density as well as for enhanced light extraction efficiency due to their high optical reflectivity ${ }^{9}$ Furthermore, after removing the native substrate, the luminescence efficiency of these vertical-cavity LEDs can be improved by thinning the optical cavity length through a backside etching process ${ }^{10}$. 
Separating the epilayers from the native substrate is a key step in the fabrication process of VTF-LEDs.

Several methods have been used for this purpose, such as laser lift-off ${ }^{11-17}$, chemical lift-off ${ }^{18-20}$, and photoelectrochemical lift-off ${ }^{21}$. However, these techniques have some limitations such as high capital cost for laser lift-off, slowness of a time-consuming process for chemical lift-off, and a low ability to reuse the substrate $^{22,23}$ for both laser and chemical lift-off. Recently, a promising transfer approach based on twodimensional crystals, namely graphene $\mathrm{e}^{24,25}$ and hexagonal boron nitride (h-BN) ${ }^{26,27}$ has emerged as an effective technology for the release and transfer of III-nitride epilayers. This approach consists of a mechanical peeling-off of epilayers from the substrate which allows a dry and fast (instantaneous) release. Helpfully, the separated surface is atomically flat allowing for an efficient transfer to rigid substrate ${ }^{28}$, and the native substrate can be reused repeatedly as a growth substrate. Moreover, when combined with selective area growth, we have demonstrated large surface crack-free planar LEDs transferred to arbitrary substrates $^{29,30}$. In addition, the use of $2 \mathrm{D}$ hBN has three key advantages. First, hBN can be grown by MOCVD in the same run and same growth conditions as the LEDs structures which is a very important characteristic. Second, it is an easy and low-cost approach to obtain LEDs membranes and other III-N devices. The third point is that using 2D crystal with no free dangling bonds on the surface gives rise to a new mechanism of growth called van der Waals (vdWs) epitaxy. With vdWs epitaxy the GaN buffer layer has less residual stress because it is weakly bonded to the $2 \mathrm{D}$ layer ${ }^{31}$. In addition, a low temperature epigrowth to move from 3D to 2D GaN buffer layer is not needed, and a $0.5-\mu m$-thick buffer layer instead of 3-4 $\mu m$ for standard epitaxy on sapphire is sufficient. 
In this work, we report a new h-BN enabled approach which allows for the monolithic fabrication of crack-free vertical LED arrays on large-area free-standing membranes. This process is enabled by the use of a thick layer of electroplated copper deposited on top of the LED structure which (i) adds rigidity to the structure to prevent crack generation, (ii) acts as back mirror and heat sink, and (iii) enables, when combined with moderate thermal treatment, the self-lift-off and transfer (SLOT) of LED structures from sapphire. We report free-standing arrays of several tens of VTF-LEDs on 30- $\mu$ m-thick copper membranes and report characterization of their electro-optical performance. The SLOT process provides a solution for the fabrication of low cost, scalable, crack-free, and highly reproducible free-standing arrays of LEDs with centimeter-sized area.

(a)

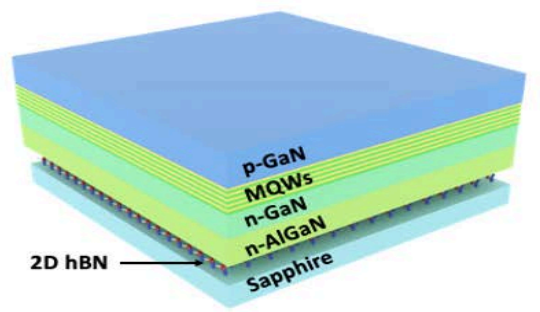

(b)

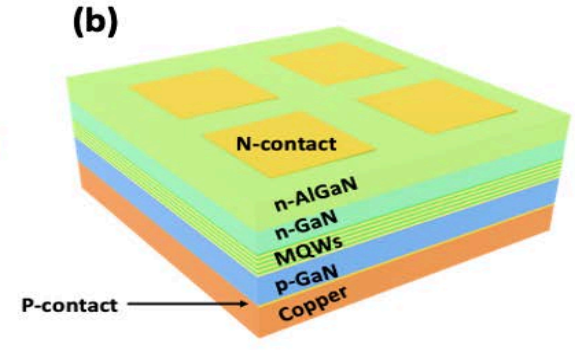

(c)

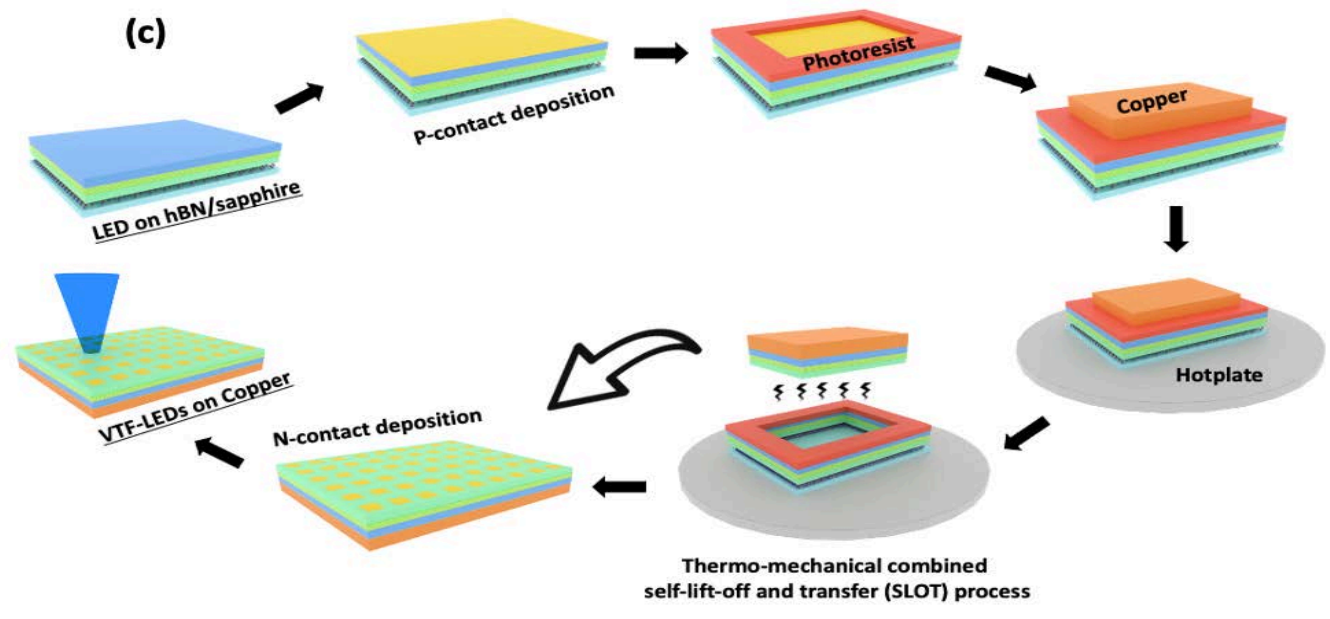

Figure 1. Schematics of (a) LEDs structure grown on hBN/sapphire, (b) VTF-LEDs on copper fabricated by SLOT process and (c) different steps of the SLOT process for fabrication of VTF-LEDs.

\section{Materials and methods}


After the growth of a 3-nm-thick h-BN layer on 2-inch sapphire substrate ${ }^{32}$, an intermediate layer of n$\operatorname{AlGaN}(0.3 \mu \mathrm{m})$ with an $\mathrm{Al}$ mole fraction of $14 \%$ was grown to act as a nucleation layer, followed by a GaN-based LED heterostructure consisting of a silicon-doped n-GaN $(0.3 \mu \mathrm{m}), 5 \mathrm{InGaN} / \mathrm{GaN}$ MQWs and a magnesium doped p-GaN region $(0.2 \mu \mathrm{m})$. The $5 \mathrm{MQWs}$ structure consists of 12 -nm-thick GaN barrier layers and 2.5-nm-thick InGaN QW layers with an In mole fraction of 15\% to get light emission in the blue (targeted wavelength is around $460 \mathrm{~nm}$ and has been confirmed with cathodoluminescence).

The electron and hole carrier concentrations in the Si- and $\mathrm{Mg}$-doped $\mathrm{GaN}$ layers are 5 x $10^{18}$ and $1 \mathrm{x}$ $10^{17} \mathrm{~cm}^{-3}$, respectively. The LED structures before and after the SLOT fabrication process are given in Figure 1a and 1b, respectively, and the SLOT fabrication process of VTF-LEDs is schematically summarized in Figure 1c. First, a Ni $(20 \mathrm{~nm}) / \mathrm{Au}(20 \mathrm{~nm})$ bilayer is deposited on top of the LED epilayer to act as a p-type electrical contact. This contact is then annealed at $600^{\circ} \mathrm{C}$ under $\mathrm{O}_{2}$ atmosphere for $60 \mathrm{~s}$ and an additional $\mathrm{Ti}(5 \mathrm{~nm}) / \mathrm{Au}(20 \mathrm{~nm})$ bilayer is deposited. The Ti layer helps to improve the adhesion of the subsequent metal layers while the Au layer acts as a seed for subsequent copper deposition as well as back mirror to enhance the reflection of the light emitted by the quantum wells. These metal layers are deposited by e-beam evaporation. Then, a few $\mathrm{cm}^{2}$ area is patterned by a photoresist (SPR 220-3) via a standard photolithography-based process, followed by an $\mathrm{O}_{2}$ plasma cleaning to remove any remaining traces of photoresist from the developed area. Then, a 30- $\mu$ m-thick layer of copper is electroplated using an acid-free solution containing dilute $\mathrm{CuSO}_{4}$ to avoid delamination of the epilayers grown on h-BN.

Thinner layers of $\mathrm{Cu}$ resulted in the free-membrane wrapping around itself generating cracks and making it hard to manipulate for further process steps. 30- $\mu$ m-thick $\mathrm{Cu}$ layer was shown to be thick enough to rigidify the structure while maintaining it flexible and result in reproducible flat and crack-free flexible membranes. The sheet resistance of the $30-\mu \mathrm{m}$-thick electroplated copper was found equal to $3 \mathrm{~m} \Omega$ per square. This value is one order of magnitude larger than values reported in the literature ${ }^{33}$. This could be explained by the fact that we used a sulfuric acid-free solution for the electroplating which generally lead 
to higher resistivity of the copper layer due to the formation of $\mathrm{CuOH}$ complexes. The release of the LED structure from the sapphire native substrate is achieved with a thermal treatment at $100^{\circ} \mathrm{C}$ for 1 hour. During this step, the whole few $\mathrm{cm}^{2}$ targeted area of the functional layers is spontaneously released from the sapphire substrate owing to shear stress at the interface between the copper layer and the III-N epilayers due to the difference of thermal expansion coefficients. This shear stress is large enough to break the van der Waals bonding of h-BN to sapphire. Extended analysis of thermal stresses generated in the layers and experimental details are given in Section 3 in the Supporting Information. From the AFM image (Section 1 in the Supporting Information), the resulting n-AlGaN surface is atomically flat with an r.m.s roughness of $0.65 \mathrm{~nm}$ obtained over a 10 x $10 \mu \mathrm{m}$ area. The High-angle annular dark field scanning transmission electron microscope (HAADF-STEM) images of the same LED structure before and after lift-off are presented in Section 1 in the Supporting Information, showing that the SLOT process does not introduce any degradation of the crystalline quality of the epilayers.

(a)

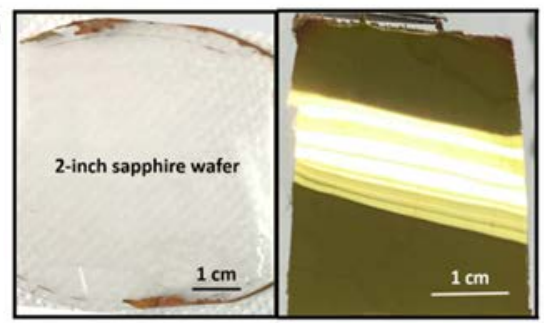

(c) (b)

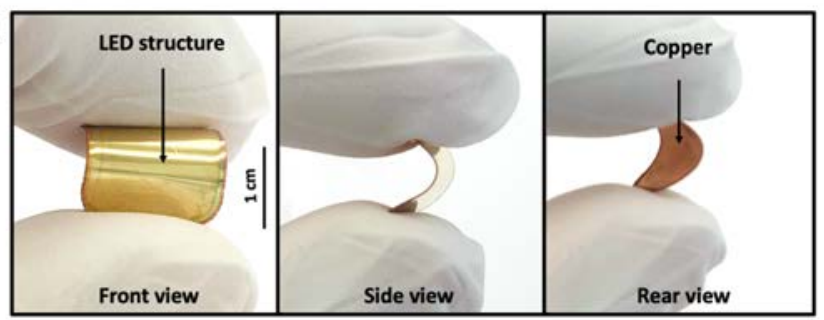

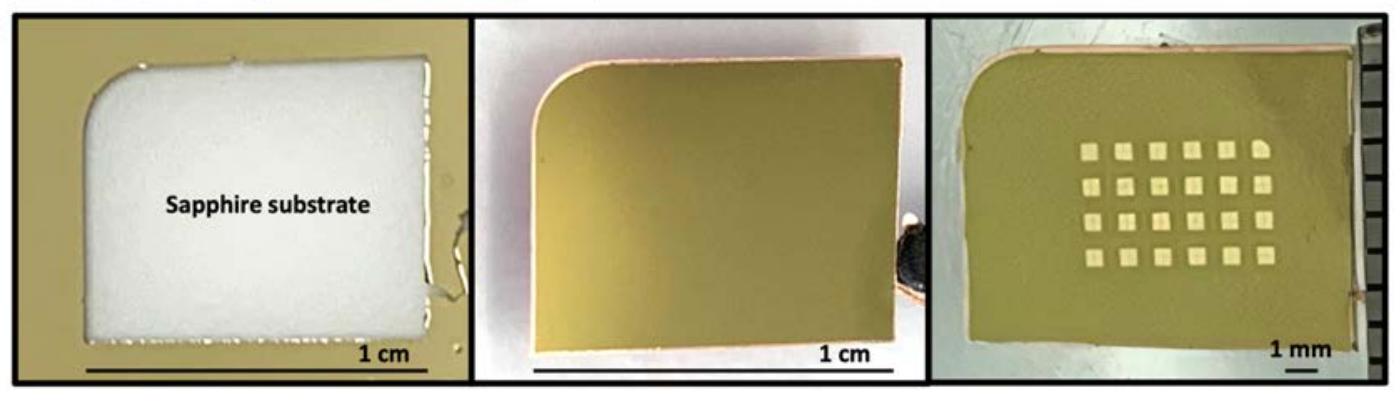

Figure 2. (a) Photographs of $4 \times 3 \mathrm{~cm}^{2}$ LED membrane and the corresponding sapphire substrate after the membrane release using the SLOT process (the bright stripe on the membrane corresponds to the reflection of the ceiling neon lamp in the cleanroom). (b) Photographs of a 1-cm2 membrane, highlighting its bending possibility. (c) Photographs of a $1-\mathrm{cm}^{2}$ released membrane on which an array of $0.5 \times 0.5 \mathrm{~mm}^{2}$ VTF-LEDs is fabricated. 
After a 10 min $\mathrm{HCl}$ treatment to remove native oxides from the exposed n-AlGaN layer, a 30-nm-thick Au layer is deposited by e-beam evaporation using a stainless-steel shadow mask with squares of various size $\left(0.25 \mathrm{~mm}^{2}\right.$ to $\left.4 \mathrm{~mm}^{2}\right)$ on the $\mathrm{n}-\mathrm{AlGaN}$ layer to form the $\mathrm{n}$-contact. This contact is not annealed to avoid inducing cracks in the free-standing LED membrane due to the high thermal expansion of the copper layer. The VTF-LED membranes obtained are then bonded to a thick aluminum holder using silver paste for further electro-optical characterizations. Electrical and electro-optical characterization was conducted on the fabricated VTF-LEDs through pulsed (1 ms duration, duty cycle of $1 \%$ ) current-voltage measurements. Optical power was measured by a Si photodiode placed, due to the electric probes size, 2 $\mathrm{cm}$ above the device under test, and thus capturing a small amount of the light emitted from the LED under test.

\section{Results}

Figure 2a shows photographs of a $4 \times 3 \mathrm{~cm}^{2}$ released LED membrane and the corresponding sapphire substrate after the membrane release using the SLOT process. Photographs of a $1-\mathrm{cm}^{2}$ membrane, highlighting its flexibility as well as a membrane on which an array of $24,0.5 \times 0.5 \mathrm{~mm}^{2}$ in size, VTFLEDs are fabricated are shown in Figures $2 \mathrm{~b}$ and $2 \mathrm{c}$, respectively. In parallel, lateral LEDs grown on $\mathrm{h}-$ $\mathrm{BN} / \mathrm{Al}_{2} \mathrm{O}_{3}$ template (using the same growth parameters) were fabricated to compare the performances of both types of devices.

Electrical and electro-optical characterizations were carried out on more than 50 LEDs of size $500 \mathrm{x}$ $500 \mu \mathrm{m}^{2}$, fabricated on different membranes. All the devices exhibited a diode-like I-V curve, with threshold voltage ranging from $5 \mathrm{~V}$ to $10 \mathrm{~V}$ and series resistance ranging from $5 \Omega$ to $15 \Omega$, and light emission. We note some variation in the efficiency and homogeneity of the emission from one LED to another. As an example, Figure 3a shows a photograph image of an array of 4 VTF-LEDs connected in parallel and operated at 8V. As shown in Figure 3b, for 15 of the set of 50 VTF-LEDs, homogeneous and bright electro-luminescence was obtained, although the applied current and voltage were different for each of them. Figure 3c shows both typical current-voltage (I-V) and optical power versus current (L-I) 
characteristics VTF-LED, recorded in this set of 15 devices. When compared to a lateral LED fabricated on a same LED structure (Figure S3 in Section 2 in the Supporting Information), the VTF-LED exhibits a higher threshold voltage (around 9V, against 2.5V for the lateral LED), lower series resistance $(5 \Omega$, against $12 \Omega$ for the lateral LED) and higher leakage current $(1.7 \mathrm{~mA}$ at $-3 \mathrm{~V}$, against $0.4 \mathrm{~mA}$ for the lateral LED). We also remark that the VTF-LED exhibits a linear increase of the optical power with the current injection increase up to $500 \mathrm{~mA}$ (limit of our equipment), without any saturation as in the case of the lateral LED for injected current larger than $200 \mathrm{~mA}$ for which current crowding and associated heating effect begin to degrade the LED efficiency. This heating effect is greatly reduced thanks to better current

(a)

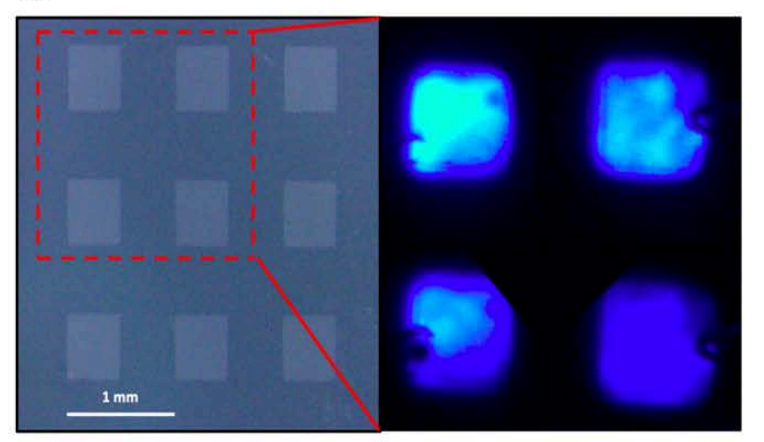

(c)

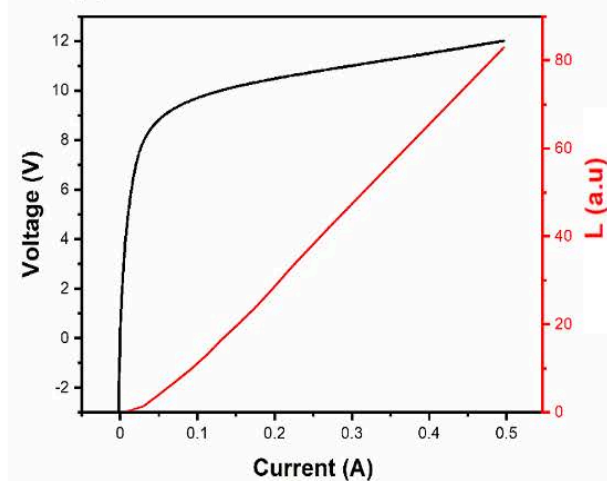

(d)

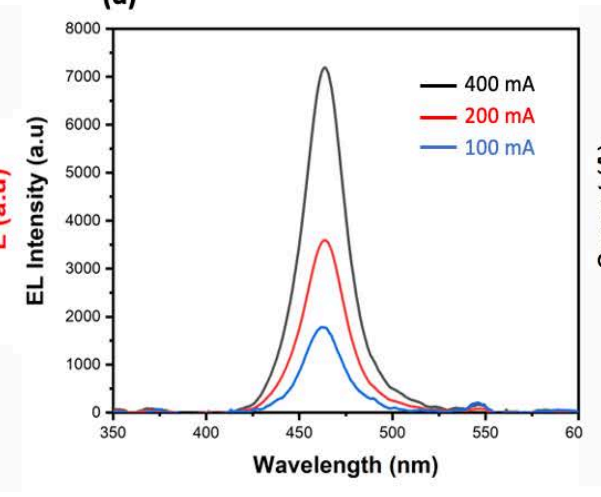

(b)

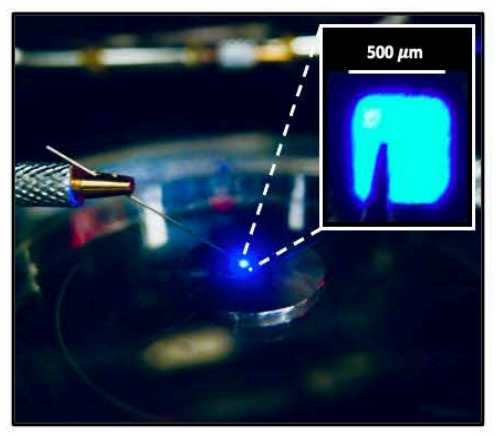

(e)

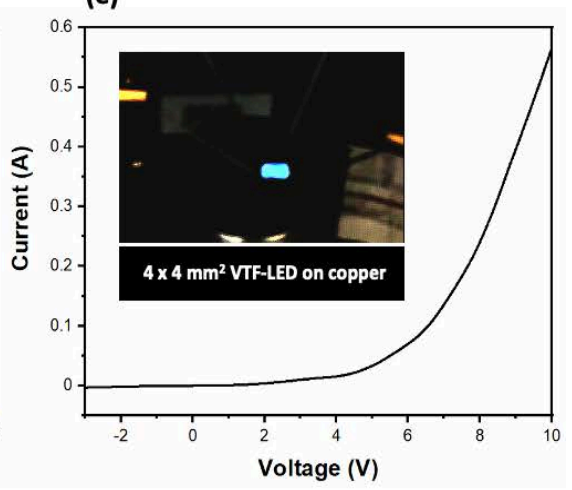

Figure 3. (a) Electroluminescence image of 4 VTF-LEDs connected in parallel and operated at 8V.

(b) Photographs of a homogeneous VTF-LED operated at $300 \mathrm{~mA}$. (c) Current-voltage and optical power versus current characteristics of the VTF-LED in (b). (d) Electro-luminescence spectra of the VTF-LED for different levels of injected current. (e) Current-voltage of a large size (4 mm x 4 $\mathrm{mm}$ ) VTF-LED. Inset is a photograph of this LED operated at $500 \mathrm{~mA}$. 
injection in the vertical structure and to the copper substrate which acts as a heat sink permitting good thermal dissipation in the case of the VTF-LED, as it has been reported by Horng et al. ${ }^{4}$.

Figure 3d shows the electro-luminescence spectra of the VTF-LEDs for different levels of injected current. The light-emission peaks are at a wavelength of $462 \mathrm{~nm}$ and are independent of the value of the injected current. As reported by Trindade et al. ${ }^{34}$, the temperature increase due to the increase of current injection should lead to a red shift whereas the reduced quantum confined stark effect (QCSE) while increasing the current injection should lead to a blue shift. In the case of these VTF-LEDs on copper, the absence of a red shift could be attributed to the high thermal dissipation capability of the copper substrate, whereas the absence of a blue shift could be attributed to the relaxation of residual compressive strain in the GaN-based films grown on h-BN, that diminishes the QCSE in the quantum wells as reported by Liu, $\mathrm{F}$ et al. ${ }^{31}$. Finally, Figure $3 \mathrm{e}$ shows both the I-V characteristic and a photograph of a large size (4 $\mathrm{mm} \times 4$ $\mathrm{mm}$ ) VTF-LED operated at a current of $500 \mathrm{~mA}$. As deduced from the I-V curve, the voltage threshold and series resistance are $6.2 \mathrm{~V}$ and $6.5 \Omega$, respectively. Rather homogeneous (without dark regions) electro-luminescence is observed, consistent with the absence of cracks in the membrane.

The results presented above demonstrate the proof of concept of an h-BN enabled SLOT process for the fabrication of VTF-LEDs. These results show that the thermo-mechanical SLOT process allows for large-area and crack-free release of III-Nitride epilayers and fabrication of VTF-LEDs arrays. This approach based on self-detachment of the epilayer from the substrate without any external force applied by a stamp/tape simplifies a critical step of the process fabrication and hence minimize the risk of cracks generation for large surface devices leading to a highly reproducible and controllable process. Also, since the membrane is only $30-\mu \mathrm{m}$ thick, the fabricated VTF-LEDs could be folded, cut and stuck to different surfaces.

Nevertheless, the n-contact of these structures is realized on the n-AlGaN layer on h-BN which, as shown in our previous work ${ }^{30}$, has lower crystalline quality than the $\mathrm{n}-\mathrm{GaN}$ buffer near the MQWs. Thus, strong carrier recombination process can occur at the grain boundaries of the AlGaN layer. This could 
explain the observed high series resistance (expected values for this kind of devices are around $1 \Omega^{20}$ ) as well as the inhomogeneous light-emission from one LED to the other and within a single LED.
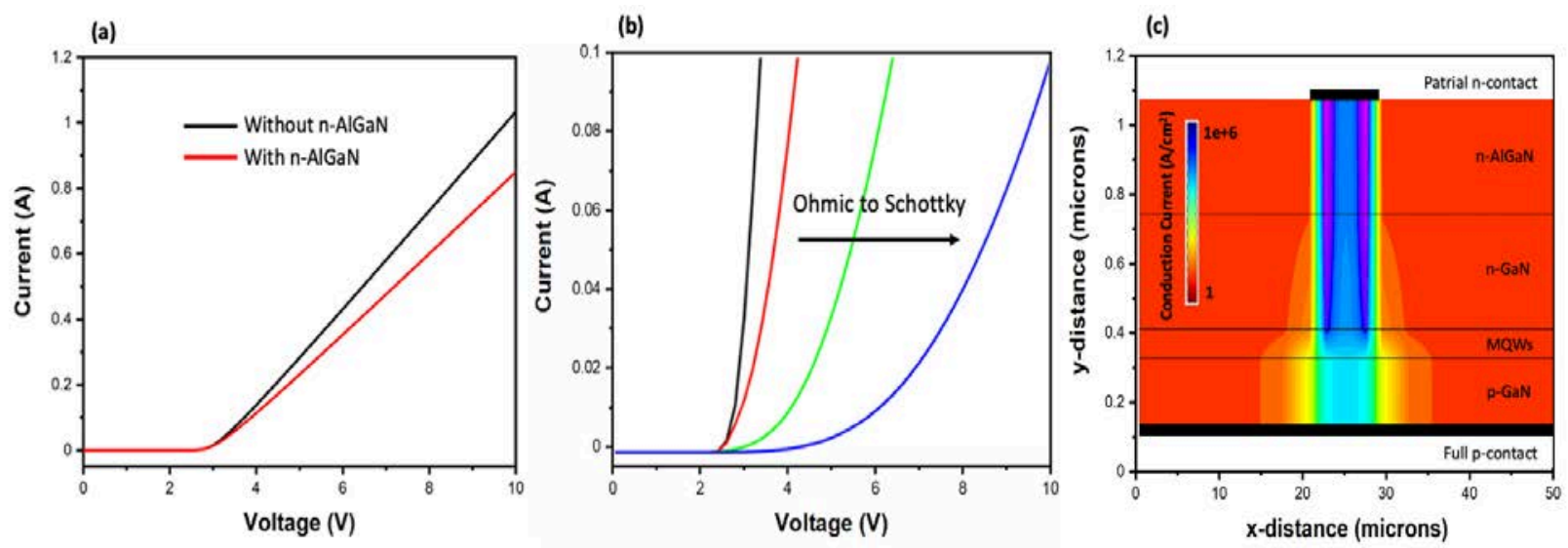

Figure 4. (a) Simulated current-voltage characteristics of VTF-LEDs with and without the nAlGaN layer. (b) Simulated effect of the Schottky n-contact on the current-voltage characteristic of VTF-LEDs. (c) Current distribution in a VTF-LED without mesa isolation.

The rather high leakage current observed in the fabricated VTF-LEDs is likely related to the absence of mesa isolation ${ }^{35}$ of single vertical devices whereas their large threshold voltage are probably due to the non-ohmic n-contact which was not annealed to avoid inducing cracks in the free-standing LED membrane. The absence of mesa isolation could also lead to some current spreading in the MQWs region resulting in lower current density and thus, reduced light-emission efficiency. These points are confirmed by electrical simulations of the VTF-LEDs, carried out using SILVACO-Atlas software to study the effect of (i) the n-AlGaN layer (considered without any defect), (ii) ohmicity of the n-contact and (iii) absence of mesa isolation. As shown in Figure 4a, the n-AlGaN layer introduces increased resistance in the structure leading to an increased series resistance of the LED, whereas, as shown in Figure 4b, the largest impact on the I-V curve comes from the non-ohmic contact which results in large increase of the threshold voltage and decrease of the current. The current spreading at the level of the quantum wells is clearly shown in Figure 4c. Thus, the AlGaN resistivity (which is probably under-estimated in the simulations which do not include the effect of low structural quality of the AlGaN layer), and the non-ohmic n-contact are the main cause of the observed high series resistance and voltage threshold whereas the current 
spreading due to non-symmetric contacts causes reduction of electro-luminescence. To remove electrical issues related to the AlGaN layer, a solution could lie in the etching of this layer. Either dry or wet AlGaN etch must be conducted on the free-standing membrane, which is challenging and requires further adaptation. Improvement in efficiency have been previously demonstrated on VTF-LEDs released through laser lift-off, where the defective undoped $\mathrm{GaN}$ buffer layer is removed through a backside etching process ${ }^{10,36}$. Work is in progress to optimize this step for the SLOT process. Optical simulation was also conducted to investigate the effect of the n-AlGaN layer on the light extraction efficiency (LEE) and far field angular emission pattern (FFP) of the VTF-LEDs. FDTD Lumerical software was used to model the structure where a dipole source is positioned at the MQW active region. The LEE and FFP were obtained by averaging results over the source position and polarization direction of the dipole to consider the incoherent, unpolarized and isotropic emission of the QWs. Figure 5a shows the LEE as a function of the optical cavity length. The n-AlGaN layer thickness is swept from 0 to $500 \mathrm{~nm}$. We can see a periodic variation of the extraction efficiency with a period of around $100 \mathrm{~nm}$, originating from interference effects altering the top surface reflection according to the AlGaN layer thickness. The LEE is shown to vary between $3.3 \%$ and $6.5 \%$. The $\mathrm{n}-\mathrm{AlGaN}$ thickness of our fabricated VTF-LEDs being around $300 \mathrm{~nm}$ is thus not optimum since the corresponding LEE is around $4.2 \%$. As it can be seen in Figure $5 \mathrm{~b}$ showing the effect of the $\mathrm{n}-\mathrm{AlGaN}$ layer thickness on the FFP, the quasi-flat pattern corresponding to the actual VTF-LED (300 nm thick AlGaN) is in a good agreement with the poorly defined light emission at the edges, seen in the inset of Figure $3 b$ and could explain also the lower measured optical emission. Slightly modifying the thickness of the n-AlGaN layer (to $340 \mathrm{~nm}$ for instance) would increase the LEE to more than $6 \%$ and result in highly directional emission of light. 


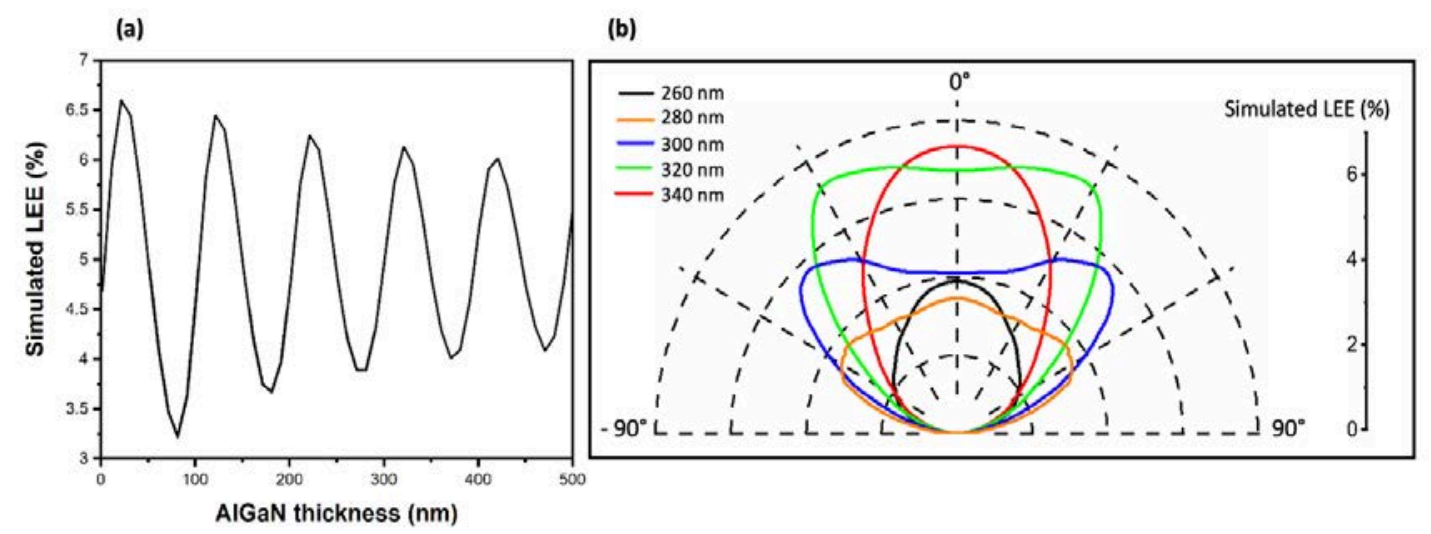

Figure 5. Simulation of the effect of $n-A l G a N$ thickness on (a) the LEE of the VTF-LEDs and (b) on their far-field angular pattern for an AlGaN thickness range from $260 \mathrm{~nm}$ to $340 \mathrm{~nm}$.

\section{Conclusion}

The results obtained in this study show that the thermo-mechanical SLOT process allows for large-area and crack-free release of III-Nitride epilayers and fabrication of VTF-LEDs arrays based on h-BN grown in the same MOVPE growth run as the epilayers. Because the process does not require any external force with tape/stamps to separate the epilayers from the substrate, this approach can take its place as additional 2D material building block enabling mechanical liftoff and transfer of epilayers. A set of ten LED membranes containing a total of 400 LEDs has been fabricated using the SLOT process and 50 LEDs have been deeply characterized in the frame of this work. Crack-free membranes on which we were able to get light emission from all of the LEDs but with dispersion in their performance have been obtained, leading to a $100 \%$ crack-free transfer yield for the 400 tested LEDs. In addition, the SLOT process at the wafer scale is possible leading to the lift-off and transfer of the LED epilayers from a full 2-inch wafer. Because the membrane is only $30-\mu \mathrm{m}$ thick, the fabricated VTF-LEDs can be folded, cut and transferred onto different surfaces. Moreover, an efficient etch of the n-AlGaN buffer layer on the free-standing membranes could enhance performances of this VTF-LEDs by decreasing the electrical series resistance as well as enhancing the LEE by modifying the optical cavity length. This work also opens new ways for the fabrication of thin-film flip-chip (TFFC) LEDs where both contacts are placed on the backside of the LED. Such a configuration ${ }^{36,37}$ allows for higher light-extraction (no metal is deposited on the emitting 
side and there is no requirement of wire-bonding to access the n-contact). TFFC-LEDs can be achieved through the SLOT process, by introducing additional process steps while the LED structure in still on the h-BN/sapphire native substrate. Besides the expected increase of the LEE, this architecture will also allow to deposit the n-contact on the good quality n-GaN buffer instead of the low-quality n-AlGaN seed layer (as for VTF-LEDs) and thus, enhance the charge carrier transport. Moreover, since for the TFFC configuration the deposition of n-contact is done while the LED structure is still on h-BN/sapphire native substrate, this contact can be annealed, which will decrease the threshold voltage and series resistance as compared to the VTF-LEDs.

\section{AUTHOR INFORMATION}

\section{Corresponding Author}

* Corresponding Author: jean-paul.salvestrini@georgiatech-metz.fr.

\section{Permanent Addresses}

$†$ SIGER Laboratory, Sidi Mohammed Ben Abdellah University, Faculty of Sciences and Technology, P. O. Box 2020, Fes, Morocco.

\section{Author Contributions}

J.P.S. and A.O. contributed to design the study and supervised the whole project. S.K. performed device fabrication and electrical measurements. S.K. R.G. and J.-P.S. achieved the lift-off and transfer of devices and their electro-optical measurements. S.K., J.-P.S., P.L.V., and A.O. wrote the manuscript. S.S., A.M., P.V. performed the epitaxial growth. K.B. and W.E.H. contributed in the optimization of the fabrication process. R.G. W.E.H. and S.K. performed electrical and optical simulations. G.P realized HAADF-STEM and EDX analysis. H. E. A. performed cathodoluminescence (CL) analysis of the LED structures and V. O. did AFM measurements of the epilayers. The manuscript was written through contributions of all authors. All authors have given approval to the final version of the manuscript. 


\section{Notes}

The authors declare no competing financial interest.

\section{ACKNOWLEDGMENT}

This study has been funded by the French National Research Agency (ANR), under the GANEX Laboratory of Excellence (Labex) and INMOST (AAP 2019) projects, and French PIA project Lorraine Université d'Excellence (Grant ANR-15-IDEX-04-LUE), as well as by the Region Grand Est in the frame of the "Chercheur d'Excellence" program.

\section{Supporting Information}

Structural and morphological characterization (AFM, HAADF-STEM and EDX); Electro-optical characterization of lateral LEDs on hBN/sapphire, Mechanical analysis of the SLOT process. REFERENCES

(1) Feezell, D.; Nakamura, S. Invention, Development, and Status of the Blue Light-Emitting Diode, the Enabler of Solid-State Lighting. Comptes Rendus Phys. 2018, 19 (3), 113-133. https://doi.org/10.1016/j.crhy.2017.12.001.

Guo, X.; Schubert, E. F. Current Crowding in GaN/InGaN Light Emitting Diodes on Insulating Substrates. J. Appl. Phys. 2001, 90 (8), 41914195. https://doi.org/10.1063/1.1403665.

Chang, S. J.; Shen, C. F.; Chen, W. S.; Ko, T. K.; Kuo, C. T.; Yu, K. H.; Shei, S. C.; Chiou, Y. Z. Nitride-Based LEDs with an Insulating SiO2 Layer underneath p-Pad Electrodes. Electrochem. Solid-State Lett. 2007, 10 (6), 175-177. https://doi.org/10.1149/1.2718392. Horng, R. H.; Chiang, C. C.; Wuu, D. S.; Hsiao, H. Y.; Lin, H. I. Enhanced Thermal Dissipation and Light Output of GaN/Sapphire LightEmitting Diode by Direct Cu Electroplating. Electrochem. Solid-State Lett. 2008, 11 (11), 300-303. https://doi.org/10.1149/1.2972992. Delmdahl, R.; Kunzer, M.; Schwarz, U. Thin Film LEDs Gaining Ground: Excimer Laser Lift-off Enables High Brightness LED Production. Laser Tech. J. 2011, 8 (3), 48-52. https://doi.org/10.1002/latj.201190027. Weisbuch, C. Review—On The Search for Efficient Solid State Light Emitters: Past, Present, Future. ECS J. Solid State Sci. Technol. 2020, 9 (1), 016022. https://doi.org/10.1149/2.0392001jss.

Wang, S.-J.; Uang, K.-M.; Chen, S.-L.; Yang, Y.-C.; Chang, S.-C.; Chen, T.-M.; Chen, C.-H.; Liou, B.-W. Use of Patterned Laser Liftoff Process and Electroplating Nickel Layer for the Fabrication of Vertical-Structured GaN-Based Light-Emitting Diodes. Appl. Phys. Lett. 2005, 87 (1), 11111. https://doi.org/10.1063/1.1993757. Tran, C. A.; Chu, C. F.; Cheng, C. C.; Liu, W. H.; Chu, J. Y.; Cheng, H. C.; Fan, F. H.; Yen, J. K.; Doan, T. High Brightness GaN Vertical Light Emitting Diodes on Metal Alloyed Substrate for General Lighting Application. J. Cryst. Growth 2007, 298 (SPEC. ISS), $722-724$. https://doi.org/10.1016/j.jcrysgro.2006.10.187. Wuu, D. S.; Hsu, S. C.; Huang, S. H.; Horng, R. H. Vertical-Conducting p-Side-up GaN/Mirror/Si Light-Emitting Diodes by Laser Lift-off and Wafer-Transfer Techniques. Phys. Status Solidi Appl. Res. 2004, 201 (12), 2699-2703. https://doi.org/10.1002/pssa.200405075. 
Green Light-Emitting Diodes. IEEE Photonics J. 2013, 5 (4), 8400407. https://doi.org/10.1109/JPHOT.2013.2274768.

Lee, H. E.; Choi, J. H.; Lee, S. H.; Jeong, M.; Shin, J. H.; Joe, D. J.; Kim, D. H.; Kim, C. W.; Park, J. H.; Lee, J. H.; Kim, D.; Shin, C. S.; Lee, K. J. Monolithic Flexible Vertical GaN Light-Emitting Diodes for a Transparent Wireless Brain Optical Stimulator. Adv. Mater. 2018, 30 (28), 1-10. https://doi.org/10.1002/adma.201800649.

Yulianto, N.; Kadja, G. T. M.; Bornemann, S.; Gahlawat, S.; Majid, N.; Triyana, K.; Abdi, F. F.; Wasisto, H. S.; Waag, A. Ultrashort Pulse Laser Lift-Off Processing of InGaN/GaN Light-Emitting Diode Chips. ACS Appl. Electron. Mater. 2021, 3 (2), 778-788.

https://doi.org/10.1021/acsaelm.0c00913.

Delmdahl, R.; Pätzel, R.; Brune, J. Large-Area Laser-Lift-Off Processing in Microelectronics. Phys. Procedia 2013, 41, 241-248. https://doi.org/https://doi.org/10.1016/j.phpro.2013.03.075.

Wasisto, H. S.; Prades, J. D.; Gülink, J.; Waag, A. Beyond Solid-State Lighting: Miniaturization, Hybrid Integration, and Applications of GaN Nano- and Micro-LEDs. Appl. Phys. Rev. 2019, 6 (4), 41315. https://doi.org/10.1063/1.5096322.

Bornemann, S.; Yulianto, N.; Spende, H.; Herbani, Y.; Prades, J. D.; Wasisto, H. S.; Waag, A. Femtosecond Laser Lift-Off with Sub-Bandgap Excitation for Production of Free-Standing GaN Light-Emitting Diode Chips. Adv. Eng. Mater. 2020, 22 (2), 1901192.

https://doi.org/https://doi.org/10.1002/adem.201901192. V. E.; Teherani, F. H.; Bove, P.; Prior, K. A.; Djebbour, Z.; McClintock, R.; Razeghi, M. Wafer-Scale Epitaxial Lift-off of Optoelectronic Grade GaN from a GaN Substrate Using a Sacrificial ZnO Interlayer. J. Phys. D. Appl. Phys. 2016, 49 (31), 315105. https://doi.org/10.1088/0022$3727 / 49 / 31 / 315105$

Hsueh, H.-H.; Ou, S.-L.; Wuu, D.-S.; Horng, R.-H. InGaN LED Fabricated on Eco-GaN Template with a Ga2O3 Sacrificial Layer for Chemical Lift-off Application. Vacuum 2015, 118, 8-12. https://doi.org/https://doi.org/10.1016/j.vacuum.2015.02.002.

Ha, J. S.; Lee, S. W.; Lee, H. J.; Lee, H. J.; Lee, S. H.; Goto, H.; Kato, T.; Fujii, K.; Cho, M. W.; Yao, T. The Fabrication of Vertical LightEmitting Diodes Using Chemical Lift-off Process. IEEE Photonics Technol. Lett. 2008, 20 (3), 175-177.

https://doi.org/10.1109/LPT.2007.912491.

Hwang, D.; Yonkee, B. P.; Addin, B. S.; Farrell, R. M.; Nakamura, S.; Speck, J. S.; DenBaars, S. Photoelectrochemical Liftoff of LEDs Grown on Freestanding C-Plane GaN Substrates. Opt. Express 2016, 24 (20), 22875. https://doi.org/10.1364/oe.24.022875.

Wong, W. S.; Sands, T.; Cheung, N. W. Damage-Free Separation of GaN Thin Films from Sapphire Substrates. Appl. Phys. Lett. 1998, 72 (5), 599-601. https://doi.org/10.1063/1.120816.

Cho, C. Y.; Lee, S. J.; Hong, S. H.; Park, S. C.; Park, S. E.; Park, Y.; Park, S. J. Growth and Separation of High Quality GaN Epilayer from Sapphire Substrate by Lateral Epitaxial Overgrowth and Wet Chemical Etching. Appl. Phys. Express 2011, 4 (1), 11-14. https://doi.org/10.1143/APEX.4.012104. Kim, J.; Bayram, C.; Park, H.; Cheng, C. W.; Dimitrakopoulos, C.; Ott, J. A.; Reuter, K. B.; Bedell, S. W.; Sadana, D. K. Principle of Direct van Der Waals Epitaxy of Single-Crystalline Films on Epitaxial Graphene. Nat. Commun. 2014, 5, 1-7. https://doi.org/10.1038/ncomms5836. 330 (6004), 655-657. https://doi.org/10.1126/science.1195403. 
Devices. Nature 2012, 484 (7393), 223-227. https://doi.org/10.1038/nature10970.

Ayari, T.; Sundaram, S.; Li, X.; Alam, S.; Bishop, C.; El Huni, W.; Jordan, M. B.; Halfaya, Y.; Gautier, S.; Voss, P. L.; Salvestrini, J. P.;

Ougazzaden, A. Heterogeneous Integration of Thin-Film InGaN-Based Solar Cells on Foreign Substrates with Enhanced Performance. ACS

Photonics 2018, 5 (8), 3003-3008. https://doi.org/10.1021/acsphotonics.8b00663.

Ayari, T.; Sundaram, S.; Li, X.; El Gmili, Y.; Voss, P. L.; Salvestrini, J. P.; Ougazzaden, A. Wafer-Scale Controlled Exfoliation of Metal Organic Vapor Phase Epitaxy Grown $\quad$ InGaN/GaN Multi Quantum Well Structures Using Low-Tack Two-Dimensional Layered h-BN. Appl. Phys. Lett. 2016, 108 (17), 171106. https://doi.org/10.1063/1.4948260.

Ayari, T.; Sundaram, S.; Bishop, C.; Mballo, A.; Vuong, P.; Halfaya, Y.; Karrakchou, S.; Gautier, S.; Voss, P. L.; Salvestrini, J. P.; Ougazzaden, A. Novel Scalable Transfer Approach for Discrete III-Nitride Devices Using Wafer-Scale Patterned h-BN/Sapphire Substrate for Pick-and-Place Applications. Adv. Mater. Technol. 2019, 4 (10), 1900164. https://doi.org/https://doi.org/10.1002/admt.201900164.

Karrakchou, S.; Sundaram, S.; Ayari, T.; Mballo, A.; Vuong, P.; Srivastava, A.; Gujrati, R.; Ahaitouf, A.; Patriarche, G. Effectiveness of Selective Area Growth Using van Der Waals h - BN Layer for Crack - Free Transfer of Large - Size III - N Devices onto Arbitrary Substrates. Sci. Rep. 2020, 1-9. https://doi.org/10.1038/s41598-020-77681-z. Liu, F.; Yu, Y.; Zhang, Y.; Rong, X.; Wang, T.; Zheng, X.; Sheng, B.; Yang, L.; Wei, J.; Wang, X.; Li, X.; Yang, X.; Xu, F.; Qin, Z.; Zhang, Z.; Shen, B.; Wang, X. Hexagonal BN-Assisted Epitaxy of Strain Released GaN Films for True Green Light-Emitting Diodes. Adv. Sci. 2020, 7 (21), 1-8. https://doi.org/10.1002/advs.202000917. Li, X.; Sundaram, S.; El Gmili, Y.; Ayari, T.; Puybaret, R.; Patriarche, G.; Voss, P. L.; Salvestrini, J. P.; Ougazzaden, A. Large-Area TwoDimensional Layered Hexagonal Boron Nitride Grown on Sapphire by Metalorganic Vapor Phase Epitaxy. Cryst. Growth Des. 2016, 16 (6), 3409-3415. https://doi.org/10.1021/acs.cgd.6b00398. Teh, W. H.; Koh, L. T.; Chen, S. M.; Xie, J.; Li, C. Y.; Foo, P. D. Study of Microstructure and Resistivity Evolution for Electroplated Copper Films at Near-Room Temperature. Microelectronics J. 2001, 32 (7), 579-585. https://doi.org/https://doi.org/10.1016/S0026-2692(01)00035-0. Trindade, A. J.; Guilhabert, B.; Xie, E. Y.; Ferreira, R.; McKendry, J. J. D.; Zhu, D.; Laurand, N.; Gu, E.; Wallis, D. J.; Watson, I. M.; Humphreys, C. J.; Dawson, M. D. Heterogeneous Integration of Gallium Nitride Light-Emitting Diodes on Diamond and Silica by Transfer Printing. Opt. Express 2015, 23 (7), 9329. https://doi.org/10.1364/oe.23.009329.

Fujii, K.; Lee, S.; Ha, J. S.; Lee, H. J.; Lee, H. J.; Lee, S. H.; Kato, T.; Cho, M. W.; Yao, T. Leakage Current Improvement of Nitride-Based Light Emitting Diodes Using CrN Buffer Layer and Its Vertical Type Application by Chemical Lift-off Process. Appl. Phys. Lett. 2009, 94 (24), $92-95$. https://doi.org/10.1063/1.3155422.

Hu, X. L.; Qi, Z. Y.; Wang, H.; Zhang, X. C. Performance of InGaN-Based Thin-Film LEDs with Flip-Chip Configuration and Concavely Patterned Surface Fabricated on Electroplating Metallic Substrate. IEEE Photonics J. 2016, 8 (1), 1-8. https://doi.org/10.1109/JPHOT.2016.2516911. 
For Table of Contents Use Only

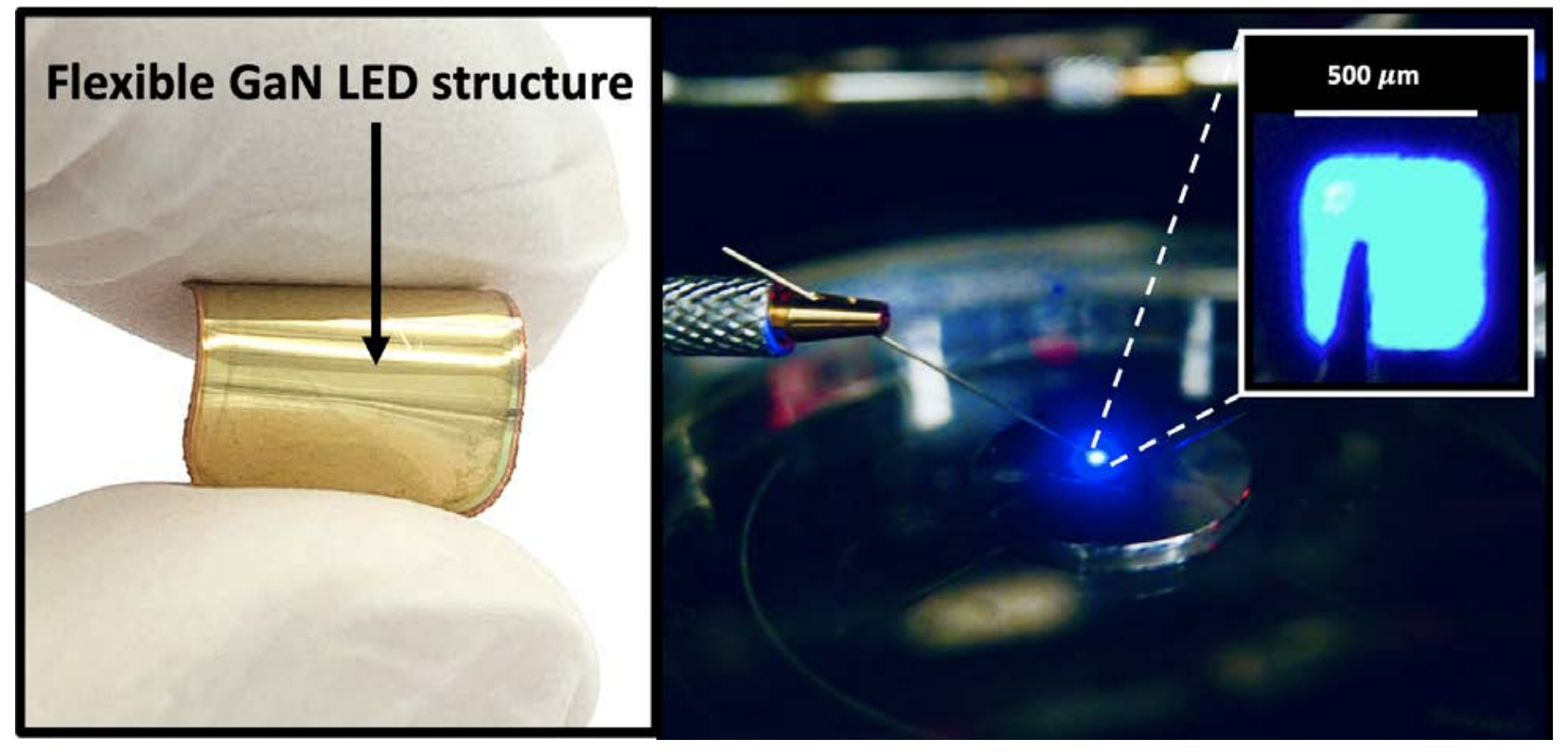

